Kragujevac Journal of Mathematics

Volume 44(1) (2020), Pages 89-100.

\title{
A NEW CLASS OF LAGUERRE-BASED GENERALIZED HERMITE-EULER POLYNOMIALS AND ITS PROPERTIES
}

\author{
N. U. KHAN ${ }^{1}$, T. USMAN ${ }^{2}$, AND W. A. KHAN ${ }^{3}$
}

\begin{abstract}
The special polynomials of more than one variable provide new means of analysis for the solutions of a wide class of partial differential equations often encountered in physical problems. Motivated by their importance and potential for applications in a variety of research fields, recently, numerous polynomials and their extensions have been introduced and investigated. In this paper, we introduce a new family of Laguerre-based generalized Hermite-Euler polynomials, which are related to the Hermite, Laguerre and Euler polynomials and numbers. The results presented in this paper are based upon the theory of the generating functions. We derive summation formulas and related bilateral series associated with the newly introduced generating function. We also point out that the results presented here, being very general, can be specialized to give many known and new identities and formulas involving relatively simple numbers and polynomials.
\end{abstract}

\section{INTRODUCTION}

The generating function of the two variable Laguerre polynomials (2-VLP) $\mathrm{E}_{n}(x, y)$ [5] is defined by

$$
\frac{1}{(1-y t)} \exp \left(\frac{-x t}{1-y t}\right)=\sum_{n=0}^{\infty} Ł_{n}(x, y) t^{n}, \quad|y t|<1,
$$

which is equivalently [6] given by

$$
\exp (y t) C_{0}(x t)=\sum_{n=0}^{\infty} \mathrm{七}_{n}(x, y) \frac{t^{n}}{n !}
$$

Key words and phrases. Hermite polynomials, Laguerre polynomials, generalized Euler polynomials, Laguerre-based generalized Hermite-Euler polynomials, summation formulae, bilateral series.

2010 Mathematics Subject Classification. Primary: 11B68, 33C45, 33C90. Secondary: 33C05.

DOI 10.46793/KgJMat2001.089K

Received: December 17, 2017.

Accepted: February 02, 2018. 
where $C_{0}(x)$ denotes the $0^{\text {th }}$ order Tricomi function. The $n^{\text {th }}$ order Tricomi functions $C_{n}(x)$ are defined as:

$$
C_{n}(x)=\sum_{r=0}^{\infty} \frac{(-1)^{r} x^{r}}{r !(n+r) !}, \quad n \in \mathbb{N}_{0},
$$

with the following generating function:

$$
\exp \left(t-\frac{x}{t}\right)=\sum_{n=-\infty}^{\infty} C_{n}(x) t^{n}
$$

for $t \neq 0$ and for all finite $x$.

The Tricomi functions $C_{n}(x)$ are characterized by the following link with the Bessel function $J_{n}(x)$ :

$$
C_{n}(x)=x^{-\frac{n}{2}} J_{n}(2 \sqrt{x})
$$

From equations (1.1) and (1.2), we obtain

$$
L_{n}(x, y)=n ! \sum_{s=0}^{n} \frac{(-1)^{s} x^{s} y^{n-s}}{(s !)^{2}(n-s) !}=y^{n} L_{n}\left(\frac{x}{y}\right) .
$$

Thus, we have

$$
\mathrm{Ł}_{n}(x, 0)=\frac{(-1)^{n} x^{n}}{n !}, \quad \mathrm{E}_{n}(0, y)=y^{n}, \quad \mathrm{七}_{n}(x, 1)=\mathrm{七}_{n}(x),
$$

where $L_{n}(x)$ are the ordinary Laguerre polynomials [1].

The 2-variable Hermite Kampé de Fériet polynomials (2VHKdFP) $H_{n}(x, y)[2,4]$ are defined as:

$$
H_{n}(x, y)=n ! \sum_{r=0}^{\left[\frac{n}{2}\right]} \frac{y^{r} x^{n-2 r}}{r !(n-2 r) !},
$$

and is supported by the following generating function:

$$
e^{x t+y t^{2}}=\sum_{n=0}^{\infty} H_{n}(x, y) \frac{t^{n}}{n !} .
$$

When $y=-1$ and $x$ is replaced by $2 x,(1.3)$ reduce to the ordinary Hermite polynomials $H_{n}(x)$ (see [2]).

Currently, Dattoli et al. ([8], p. 241) introduced the 3-variable Laguerre-Hermite polynomials (3VLHP) ${ }_{L} H_{n}(x, y, z)$ which is defined as:

$$
{ }_{L} H_{n}(x, y, z)=n ! \sum_{k=0}^{[n / 2]} \frac{z^{k} L_{n-2 k}(x, y)}{k !(n-2 k) !} .
$$

The 3-variable Laguerre-Hermite polynomials $(3 \mathrm{VLHP}){ }_{L} H_{n}(x, y, z)$ of the following generating function:

$$
\frac{1}{(1-z t)} \exp \left(\frac{-x t}{1-z t}+\frac{y t^{2}}{1-z t^{2}}\right)=\sum_{n=0}^{\infty}{ }_{L} H_{n}(x, y, z) t^{n}
$$


equivalent to

$$
\exp \left(y t+z t^{2}\right) C_{0}(x t)=\sum_{n=0}^{\infty}{ }_{L} H_{n}(x, y, z) \frac{t^{n}}{n !} .
$$

It is clear that

$$
\begin{gathered}
{ }_{L} H_{n}\left(x, y,-\frac{1}{2}\right)={ }_{L} H_{n}(x, y), \\
{ }_{L} H_{n}(x, 1,-1)={ }_{L} H_{n}(x),
\end{gathered}
$$

where ${ }_{L} H_{n}(x, y)$ denotes the 2-variable Laguerre-Hermite polynomials (2VLHP) (see [6]) and ${ }_{L} H_{n}(x)$ denotes the Laguerre-Hermite polynomials (LHP) (see [7]), respectively.

The generalized Bernoulli $B_{n}^{(\alpha)}(x)$, Euler $E_{n}^{(\alpha)}(x)$ and Genocchi $G_{n}^{(\alpha)}(x)$ polynomials of order $\alpha \in \mathbb{C}$, each of degree $\mathrm{n}$ are defined respectively, by the following generating functions (see $[3,5,10,18,20]$ ):

$$
\begin{array}{ll}
\left(\frac{t}{e^{t}-1}\right)^{\alpha} e^{x t}=\sum_{n=0}^{\infty} B_{n}^{(\alpha)}(x) \frac{t^{n}}{n !}, & |t|<2 \pi, 1^{\alpha}=1, \\
\left(\frac{2}{e^{t}+1}\right)^{\alpha} e^{x t}=\sum_{n=0}^{\infty} E_{n}^{(\alpha)}(x) \frac{t^{n}}{n !}, & |t|<\pi, 1^{\alpha}=1, \\
\left(\frac{2 t}{e^{t}+1}\right)^{\alpha} e^{x t}=\sum_{n=0}^{\infty} G_{n}^{(\alpha)}(x) \frac{t^{n}}{n !}, & |t|<\pi, 1^{\alpha}=1 .
\end{array}
$$

It is easy to see that

$$
B_{n}^{(1)}(x)=B_{n}(x), \quad E_{n}^{(1)}(x)=E_{n}(x), \quad G_{n}^{(1)}(x)=G_{n}(x) .
$$

Recently, Kurt [17] introduced and investigated the generalized Euler polynomials $E^{[\alpha, m-1]}(x), m \in \mathbb{N}$ defined in a suitable neighborhood of $t=0$, by means of the generating function:

$$
\left(\frac{2^{m}}{e^{t}+\sum_{h=0}^{m-1} \frac{t^{h}}{h !}}\right)^{\alpha} e^{x t}=\sum_{n=0}^{\infty} E_{n}^{[\alpha, m-1]}(x) \frac{t^{n}}{n !} .
$$

Furthermore, we recall here an interesting (partly bilateral and partly unilateral) generating function for $L_{n}^{(\alpha)}(x)$ due to Exton [9] in the following modified form (see Pathan and Yasmeen [19], Pathan and Bin-Saad [22]):

$$
\exp \left(y+z-\frac{x z}{y}\right)=\sum_{m=-\infty}^{\infty} \sum_{n=m^{*}}^{\infty} \frac{L_{n}^{(m)}(x) y^{m} z^{n}}{(m+n) !},
$$

where $m^{*}=\max \{0,-m\}, m \in \mathbb{Z}:=\{0, \pm 1, \ldots\}$.

The reason of interest for this family of Laguerre-based generalized Hermite-Euler polynomials is due to their intrinsic mathematical importance and to the fact that these polynomials are shown to be natural solutions of a particular set of partial 
differential equations which often appears in the treatment of radiation physics problems such as the electromagnetic wave propagation and quantum beam life-time in storage rings. Motivated by their importance and potential for applications in certain problems in number theory, combinatorics, classical and numerical analysis and other field of applied mathematics, a number of certain number and polynomials, and their generalizations have recently been extensively investigated.

The organization of this paper is given as follows. In Section 2, we introduce a new class of generalized Laguerre-based Hermite-Euler polynomials ${ }_{L} H^{E_{n}{ }^{[\alpha, m-1]}}(x, y, z)$ and develop some elementary properties by using generating functions for the numbers. In Section 3, we derive the summation formulae for these generalized polynomials by using different analytical means on their respective generating functions. In Section 4, we establish generating function for Laguerre-based Hermite-Euler polynomials involving bilateral series, some of whose special cases are also presented. Relevant connections of some results presented here with those involving simpler known partly unilateral and partly bilateral representations are also indicated.

\section{A New Class of Laguerre-Based Generalized Hermite-Euler POLYNOMIALS}

In this section, we introduce the Laguerre-based generalized Hermite-Euler polynomials ${ }_{L} H^{E_{n}{ }^{[\alpha, m-1]}}(x, y, z)$, for a real or complex parameter $\alpha$ defined by means of the generating function in a suitable neighborhood of $t=0$ :

$$
\left(\frac{2^{m}}{e^{t}+\sum_{h=0}^{m-1} \frac{t^{h}}{h !}}\right)^{\alpha} e^{y t+z t^{2}} C_{0}(x t)=\sum_{n=0}^{\infty}{ }_{L} H^{E_{n}[\alpha, m-1]}(x, y, z) \frac{t^{n}}{n !},
$$

so that

$$
{ }_{L} H^{E_{n}[\alpha, m-1]}(x, y, z)=\sum_{r=0}^{n}\left(\begin{array}{l}
n \\
r
\end{array}\right) E_{n-r}^{[\alpha, m-1]}{ }_{L} H_{r}(x, y, z) .
$$

It contain as its special cases not only generalized Euler polynomials $(1.5), E_{n}^{[\alpha, m-1]}(x)$ (see (1.4)), but also generalization of Laguerre-Hermite polynomials (see (1.5)).

Setting $m=10, z=0, y \rightarrow x, z \rightarrow y$ in (2.1), the result reduces to known result of Khan et al. [11]. Again setting $x=0, y \rightarrow x, z \rightarrow y$, the result reduces to known result of Pathan and Khan [20].

For $m=1, x=0, y \rightarrow x, z \rightarrow y$, we obtain from (2.1):

$$
\left(\frac{2}{e^{t}+1}\right)^{\alpha} e^{y t+z t^{2}}=\sum_{n=0}^{\infty}{ }_{H} E_{n}^{(\alpha)}(y, z) \frac{t^{n}}{n !}
$$

which is a generalization of the generating function of Dattoli et al. ([4], p. 386 (1.6)) in the form:

$$
\left(\frac{2}{e^{t}+1}\right) e^{x t+y t^{2}}=\sum_{n=0}^{\infty}{ }_{H} E_{n}(x, y) \frac{t^{n}}{n !}
$$


Now, here we investigate the connection between Laguerre-Hermite polynomials ${ }_{L} H_{n}(x, y, z)$ and generalized Euler numbers $E_{n}^{[\alpha, m-1]}$ which are great importance in the following theorems.

Theorem 2.1. The following formula involving Laguerre-Hermite polynomials ${ }_{L} H_{n}(x, y, z)$ and Laguerre-based generalized Hermite-Euler polynomials ${ }_{L} H^{E_{n}[\alpha, m-1]}(x, y, z)$ holds true:

$$
{ }_{L} H_{n}(x, y, z)=\frac{1}{2}\left({ }_{L} H^{E_{n}[1,1]}(x, y+1, z)+{ }_{L} H^{E_{n}{ }^{[1,1]}}(x, y, z)\right) .
$$

Proof. Consider equation (2.1), we have

$$
\begin{aligned}
e^{y t+z t^{2}} C_{0}(x t) & =\frac{e^{t}+1}{2}\left(\frac{2}{e^{t}+1}\right) e^{y t+z t^{2}} C_{0}(x t) \\
& =\frac{1}{2}\left(\left(\frac{2}{e^{t}+1}\right) e^{(y+1) t+z t^{2}} C_{0}(x t)+\left(\frac{2}{e^{t}+1}\right) e^{y t+z t^{2}} C_{0}(x t)\right) .
\end{aligned}
$$

Then by using the definition of Kampé de Fériet generalization of the LaguerreHermite polynomials ${ }_{L} H_{n}(x, y)$ and Laguerre-based Hermite-Bernoulli polynomials ${ }_{L} H^{B_{n}{ }^{[\alpha, m-1]}}(x, y, z)$, we have

$$
\sum_{n=0}^{\infty}{ }_{L} H_{n}(x, y, z) \frac{t^{n}}{n !}=\frac{1}{2} \sum_{n=0}^{\infty}\left({ }_{L} H^{E_{n}[1,1]}(x, y+1, z)+{ }_{L} H^{E_{n}[1,1]}(x, y, z)\right) \frac{t^{n}}{n !} .
$$

Finally, comparing the coefficients of $\frac{t^{n}}{n !}$ in both sides, we get (2.2).

Theorem 2.2. The following formula involving Laguerre-based generalized HermiteEuler polynomials ${ }_{L} H^{E_{n}{ }^{[\alpha, m-1]}}(x, y, z)$ holds true:

$$
{ }_{L} H^{E_{n}[\alpha+\beta, m-1]}(w, x, y, z)=\sum_{r=0}^{n}\left(\begin{array}{l}
n \\
r
\end{array}\right) E_{n-r}^{[\alpha, m-1]}(w)_{L} H^{E_{n}^{[\beta, m-1]}}(x, y, z) .
$$

Proof. By analyzing definition (2.1), we have

$$
\begin{aligned}
& \left(\frac{2^{m}}{e^{t}+\sum_{h=0}^{m-1} \frac{t^{h}}{h !}}\right)^{\alpha+\beta}\left(\exp (y+w) t+z t^{2}\right) C_{0}(x t)=\sum_{n=0}^{\infty}{ }_{L} H^{E_{n}{ }^{[\alpha+\beta, m-1]}}(x, y, z) \frac{t^{n}}{n !} \\
= & \sum_{n=0}^{\infty} E_{n}^{[\alpha, m-1]}(w) \frac{t^{n}}{n !} \sum_{r=0}^{\infty}{ }_{L} H^{E_{r}[\beta, m-1]}(x, y, z) \frac{t^{r}}{r !} .
\end{aligned}
$$

Now replacing $n$ by $n-r$ in the r.h.s. of above equation and comparing the coefficients of $\frac{t^{n}}{n !}$ in both sides, we obtain the result (2.3).

Theorem 2.3. The following formula involving Laguerre-based generalized HermiteEuler polynomials ${ }_{L} H^{E_{n}{ }^{[\alpha, m-1]}}(x, y, z)$ holds true:

$$
{ }_{L} H^{E_{n}{ }^{[\alpha, m-1]}}(x, y, z)=\sum_{r=0}^{n} E_{n-r}^{[m-1]}{ }_{L} H^{E_{n}{ }^{[\alpha-1, m-1]}}(x, y, z) .
$$


Proof. Definition (2.1) can be written as

$$
\begin{aligned}
\sum_{n=0}^{\infty}{ }_{L} H^{E_{n}[\alpha, m-1]}(x, y, z) \frac{t^{n}}{n !} & =\left(\frac{2^{m}}{e^{t}+\sum_{h=0}^{m-1} \frac{t^{h}}{h !}}\right)\left(\left(\frac{2^{m}}{e^{t}+\sum_{h=0}^{m-1} \frac{t^{h}}{h !}}\right)^{\alpha-1} \exp \left(y t+z t^{2}\right) C_{0}(x t)\right) \\
& =\sum_{n=0}^{\infty} E_{n}^{[m-1]} \frac{t^{n}}{n !} \sum_{r=0}^{\infty}{ }_{L} H^{E_{n}[\alpha-1, m-1]}(x, y, z) \frac{t^{r}}{r !} .
\end{aligned}
$$

On replacing $n$ by $n-r$ in the r.h.s. of above equation and comparing the coefficients of $\frac{t^{n}}{n !}$ in both sides, we arrive at the desired result (2.4).

\section{Summation Formulae for Laguerre-Based Generalized Hermite-Euler Polynomials}

For the derivation of implicit summation formulae involving the LHEP ${ }_{L} H^{E_{n}{ }^{[\alpha, m-1]}}(x, y, z)$ the same consideration as developed for the Hermite-Bernoulli polynomials in Pathan [20] and Khan et al. [12-16] holds as well. First, we prove the following result involving the $\operatorname{LHEP}_{L} H^{E_{n}{ }^{[\alpha, m-1]}}(x, y, z)$ by using series rearrangement techniques and considered its special case.

Theorem 3.1. The following summation formula for Laguerre-based generalized Hermite-Euler polynomials ${ }_{L} H^{E_{n}{ }^{[\alpha, m-1]}}(x, y, z)$ holds true:

$$
{ }_{L} H^{E_{q+l^{[}}[\alpha, m-1]}(x, w, z)=\sum_{n, p=0}^{q, l}\left(\begin{array}{c}
q \\
n
\end{array}\right)\left(\begin{array}{l}
l \\
p
\end{array}\right)(w-y)^{n+p} H^{E_{q+l-n-p}^{[\alpha, m-1]}}(x, y, z) .
$$

Proof. Replacing $t$ by $t+u$ in (2.1) and then using the formula ([21], p. 52(2)):

$$
\sum_{N=0}^{\infty} f(N) \frac{(x+y)^{N}}{N !}=\sum_{n, m=0}^{\infty} f(n+m) \frac{x^{n}}{n !} \frac{y^{m}}{m !},
$$

in the resultant equation, we find the following generating function for the Laguerrebased Hermite-Euler polynomials ${ }_{L} H^{E_{n}{ }^{[\alpha, m-1]}}(x, y, z)$ :

$$
\left(\frac{2^{m}}{e^{t+u}+\sum_{h=0}^{m-1} \frac{(t+u)^{h}}{h !}}\right)^{\alpha} e^{z(t+u)^{2}} C_{0}(x(t+u))=e^{-y(t+u)} \sum_{q, l=0}^{\infty}{ }_{L} H^{E_{q+l}[\alpha, m-1]}(x, y, z) \frac{t^{q}}{q !} \frac{u^{l}}{l !} .
$$

Replacing $y$ by $w$ in the above equation and equating the resultant equation to the above equation, we find

$$
\exp ((w-y)(t+u)) \sum_{q, l=0}^{\infty}{ }_{L} H^{E_{q+l}{ }^{[\alpha, m-1]}}(x, y, z) \frac{t^{q}}{q !} \frac{u^{l}}{l !}=\sum_{q, l=0}^{\infty}{ }_{L} H^{E_{q+l}[\alpha, m-1]}(x, w, z) \frac{t^{q}}{q !} \frac{u^{l}}{l !}
$$


On expanding exponential function (3.3) gives

$$
\begin{aligned}
& \sum_{N=0}^{\infty} \frac{[(w-y)(t+u)]^{N}}{N !} \sum_{q, l=0}^{\infty}{ }_{L} H^{E_{q+l}{ }^{[\alpha, m-1]}}(x, y, z) \frac{t^{q}}{q !} \frac{u^{l}}{l !} \\
= & \sum_{q, l=0}^{\infty}{ }_{L} H^{E_{q+l}{ }^{[\alpha, m-1]}}(x, w, z) \frac{t^{q}}{q !} \frac{u^{l}}{l !},
\end{aligned}
$$

which on using formula (3.2) in the first summation on the left hand side becomes

$$
\begin{aligned}
& \sum_{n, p=0}^{\infty} \frac{(w-y)^{n+p} t^{n} u^{p}}{n ! p !} \sum_{q, l=0}^{\infty}{ }_{L} H^{E_{q+l}{ }^{[\alpha, m-1]}}(x, y, z) \frac{t^{q}}{q !} \frac{u^{l}}{l !} \\
= & \sum_{q, l=0}^{\infty}{ }_{L} H^{E_{q+l}[\alpha, m-1]}(x, w, z) \frac{t^{q}}{q !} \frac{u^{l}}{l !} .
\end{aligned}
$$

Now replacing $q$ by $q-n, l$ by $l-p$ and using the lemma ([19, p. 100(1)]):

$$
\sum_{k=0}^{\infty} \sum_{n=0}^{\infty} A(n, k)=\sum_{k=0}^{\infty} \sum_{n=0}^{k} A(n, k-n)
$$

in the l.h.s. of (3.4), we find

$$
\begin{aligned}
& \sum_{q, l=0}^{\infty} \sum_{n, p=0}^{q, l} \frac{(w-y)^{n+p}}{n ! p !}{ }_{L} H^{E_{q+l-n-p}{ }^{[\alpha, m-1]}}(x, y, z) \frac{t^{q}}{(q-n) !} \frac{u^{l}}{(l-p) !} \\
= & \sum_{q, l=0}^{\infty}{ }_{L} H^{E_{q+l}[\alpha, m-1]}(x, w, z) \frac{t^{q}}{q !} \frac{u^{l}}{l !} .
\end{aligned}
$$

Finally, on equating the coefficients of the like powers of $t$ and $u$ in the above equation, we get the assertion (3.1) of Theorem 3.1.

Remark 3.1. Taking $l=0$ in assertion (3.1) of Theorem 3.1, we deduce the following consequence of Theorem 3.1.

Corollary 3.1. The following summation formula for Laguerre-based Hermite-Euler polynomials ${ }_{L} H^{E_{n}^{[\alpha, m-1]}}(x, y, z)$ holds true:

$$
{ }_{L} H^{E_{q}[\alpha, m-1]}(x, w, z)=\sum_{n=0}^{q}\left(\begin{array}{c}
q \\
n
\end{array}\right)(w-y)^{n}{ }_{L} H^{E_{q-n}[\alpha, m-1]}(x, y, z) .
$$

Remark 3.2. Replacing $w$ by $w+y$ in (3.6), we obtain

$$
{ }_{L} H^{E_{q}[\alpha, m-1]}(x, w+y, z)=\sum_{n=0}^{q}\left(\begin{array}{c}
q \\
n
\end{array}\right) w^{n}{ }_{L} H^{E_{q-n}[\alpha, m-1]}(x, y, z) .
$$

Next, we prove the following result involving the product of the Laguerre-based Hermite-Euler polynomials ${ }_{L} H^{E_{n}^{[\alpha, m-1]}}(x, y, z)$ by using series rearrangement techniques. 
Theorem 3.2. The following summation formula involving the product of Laguerrebased Hermite-Euler polynomials ${ }_{L} H^{E_{n}^{[\alpha, m-1]}}(x, y, z)$ holds true:

$$
\begin{aligned}
& { }_{L} H^{E_{n}{ }^{[\alpha, m-1]}}(x, w, u){ }_{L} H^{E_{s}{ }^{[\alpha, m-1]}}(X, W, U)=\sum_{r, k=0}^{n, s}\left(\begin{array}{l}
n \\
r
\end{array}\right)\left(\begin{array}{l}
s \\
k
\end{array}\right) H_{r}(w-y, u-z) \\
& \times{ }_{L} H^{E_{n-r}[\alpha, m-1]}(x, y, z) H_{k}(W-Y, U-Z)_{L} H^{E_{s-k}[\alpha, m-1]}(X, Y, Z) .
\end{aligned}
$$

Proof. Consider the product of the Laguerre-based Hermite-Euler polynomials generating function (2.1) in the following form:

$$
\left(\frac{2^{m}}{e^{t}+\sum_{h=0}^{m-1} \frac{t^{h}}{h !}}\right)^{\alpha} \exp \left(y t+z t^{2}\right) C_{0}(x t)\left(\frac{2^{m}}{e^{T}+\sum_{h=0}^{m-1} \frac{T^{h}}{h !}}\right)^{\alpha} \exp \left(Y T+Z T^{2}\right) C_{0}(X T)
$$

$$
=\sum_{n=0}^{\infty}{ }_{L} H^{E_{n}[\alpha, m-1]}(x, y, z) \frac{t^{n}}{n !} \sum_{s=0}^{\infty}{ }_{L} H^{E_{s}[\alpha, m-1]}(X, Y, Z) \frac{T^{s}}{s !} .
$$

Replacing $y$ by $w, z$ by $u, Y$ by $W$ and $Z$ by $U$ in (3.8) and equating the resultant equation to itself, we find

$$
\begin{aligned}
& \sum_{n=0}^{\infty} \sum_{s=0}^{\infty}{ }_{L} H^{E_{n}{ }^{[\alpha, m-1]}}(x, w, u){ }_{L} H^{E_{s}[\alpha, m-1]}(X, W, U) \frac{t^{n}}{n !} \frac{T^{s}}{s !} \\
= & \exp \left((w-y) t+(u-z) t^{2}\right) \exp \left((W-Y) T+(U-Z) T^{2}\right) \\
& \times \sum_{n=0}^{\infty} \sum_{s=0}^{\infty}{ }_{L} H^{E_{n}[\alpha, m-1]}(x, y, z){ }_{L} H^{E_{s}[\alpha, m-1]}(X, Y, Z) \frac{t^{n}}{n !} \frac{T^{s}}{s !}
\end{aligned}
$$

which on using the generating function (3.5) in the exponential on the r.h.s, becomes

$$
\begin{aligned}
& \sum_{n=0}^{\infty} \sum_{s=0}^{\infty}{ }_{L} H^{E_{n}[\alpha, m-1]}(x, w, u)_{L} H^{E_{s}{ }^{[\alpha, m-1]}}(X, W, U) \frac{t^{n}}{n !} \frac{T^{s}}{s !} \\
= & \sum_{n, r=0}^{\infty} \sum_{s, k=0}^{\infty} H_{r}(w-y, u-z)_{L} H^{E_{n}[\alpha, m-1]}(x, y, z) \frac{t^{n+r}}{n ! r !} \\
& \times H_{k}(W-Y, U-Z)_{L} H^{E_{s}[\alpha, m-1]}(X, Y, Z) \frac{T^{s+k}}{s ! k !} .
\end{aligned}
$$

Finally, replacing $n$ by $n-r$ and $s$ by $s-k$ and using equation (3.5) in the r.h.s. of the above equation and then equating the coefficients of like powers of $t$ and $T$, we get assertion (3.7) of Theorem 3.2.

Remark 3.3. Replacing $u$ by $z$ and $U$ by $Z$ in assertion (3.7) of Theorem 3.2, we deduce the the following consequence of Theorem 3.2. 
Corollary 3.2. The following summation formula involving the product of Laguerrebased Hermite-Euler polynomials ${ }_{L} H^{E_{n}^{[\alpha, m-1]}}(x, y, z)$ holds true:

$$
\begin{aligned}
& { }_{L} H^{E_{n}{ }^{[\alpha, m-1]}}(x, w, z){ }_{L} H^{E_{s}[\alpha, m-1]}(X, W, Z)=\sum_{r, k=0}^{n, s}\left(\begin{array}{l}
n \\
r
\end{array}\right)\left(\begin{array}{l}
s \\
k
\end{array}\right)(w-y)^{r} \\
& \times{ }_{L} H^{E_{n-r}[\alpha, m-1]}(x, y, z)(W-Y)^{k}{ }_{L} H^{E_{s-k}[\alpha, m-1]}(X, Y, Z) .
\end{aligned}
$$

Further, we prove the following results concerning the Laguerre-based Hermite-Euler polynomials ${ }_{L} H^{E_{n}{ }^{[\alpha, m-1]}}(x, y, z)$ with $2 \operatorname{VgLP} L_{n}(x, y)$ and the generalized Hermite Euler polynomials ${ }_{H} E_{n}^{[\alpha, m-1]}$ by using operational techniques.

Theorem 3.3. The following summation formula for Laguerre-based Hermite-Euler polynomials ${ }_{L} H^{E_{n}^{[\alpha, m-1]}}(x, y, z)$ holds true:

$$
{ }_{L} H^{E_{n}[\alpha, m-1]}(z, w, y)=\sum_{n, p=0}^{k, l}\left(\begin{array}{l}
k \\
n
\end{array}\right)\left(\begin{array}{l}
l \\
p
\end{array}\right){ }_{H} E_{l+k-n-p}^{[\alpha, m-1]}(x, y)_{q} L_{n+r}(w, z-x) .
$$

Proof. We start by a recently derived summation formula for the generalized HermiteEuler polynomials ${ }_{H} E_{n}^{[\alpha, m-1]}$ (see [18]):

$$
{ }_{H} E_{k+l}^{[\alpha, m-1]}(z, y)=\sum_{n, p=0}^{k, l}\left(\begin{array}{l}
k \\
n
\end{array}\right)\left(\begin{array}{l}
l \\
p
\end{array}\right)(z-x)^{n+p}{ }_{H} E_{l+k-n-p}^{[\alpha, m-1]}(x, y) .
$$

Operating $\exp \left(D_{w}^{-1} \frac{\delta^{q}}{\delta z^{q}}\right)$ on both sides of equation (3.10), we have

$$
\begin{aligned}
& \exp \left(D_{w}^{-1} \frac{\delta^{q}}{\delta z^{q}}\right){ }_{H} E_{k+l}^{[\alpha, m-1]}(z, y) \\
= & \sum_{n, p=0}^{k, l}\left(\begin{array}{c}
k \\
n
\end{array}\right)\left(\begin{array}{l}
l \\
p
\end{array}\right){ }_{H} E_{l+k-n-p}^{[\alpha, m-1]}(x, y) \exp \left(D_{w}^{-1} \frac{\delta^{q}}{\delta z^{q}}\right)(z-x)^{n+p} .
\end{aligned}
$$

Using the operational definitions (see [12]) in the l.h.s. and r.h.s. respectively of equation (3.11), we get assertion (3.9) of Theorem 3.3.

\section{Generating Functions for the Laguerre-Based Hermite-Euler Polynomials Involving Bilateral Series}

Let us consider the following a function:

$$
V^{(\alpha, m)}=V^{(\alpha, m)}(x, y, z, w ; s, t)=\left(\frac{2^{m}}{e^{t}+\sum_{h=0}^{m-1} \frac{t^{h}}{h !}}\right)^{\alpha} e^{s-\frac{w t}{s}+y t+z t^{2}} C_{0}(x t) .
$$

Expanding $\exp \left(s-\frac{w t}{s}\right)$ in series form and then by using (2.1), we get

$$
V^{(\alpha, m)}=\sum_{M=0}^{\infty} \frac{s^{M}}{M !} \sum_{K=0}^{\infty}\left(\frac{-w t}{s}\right)^{K} \frac{1}{K !} \sum_{N=0}^{\infty}{ }_{L} H^{E_{N}[\alpha, m-1]}(x, y, z) \frac{t^{N}}{N !} .
$$


Upon replacing the summation indices $M$ and $N$ in (4.1) by $K+N=n$ and $M-K=$ $m$, respectively and rearranging the summation series:

$$
V^{(\alpha, m)}=\sum_{m=-\infty}^{\infty} \sum_{n=m^{*}}^{\infty} s^{m} t^{n} \sum_{K=0}^{n} \frac{(-w)^{K}}{K !(m+K) !(n-K) !}{ }_{L} H^{E_{n-K}[\alpha, m-1]}(x, y, z),
$$

(which can be justified by absolute convergence of the series involved), we are led to the generating function:

$$
e^{s-\frac{w t}{s}+y t+z t^{2}} C_{0}(x t)=\sum_{m=-\infty}^{\infty} \sum_{n=m^{*}}^{\infty} s^{m} t^{n} \sum_{K=0}^{n} \frac{(-w)^{K}}{K !(m+K) !(n-K) !}{ }^{L} H_{n-K}(x, y, z) .
$$

Some special cases of the result (4.2) are as follows.

(i) Setting $x=0, y=1$ and using $L_{n}(0,1)=1$, (4.2) reduces to

$$
\begin{aligned}
& \left(\frac{2^{m}}{e^{t}+\sum_{h=0}^{m-1} \frac{t^{h}}{h !}}\right)^{\alpha} e^{s-\frac{w t}{s}+z t^{2}} \\
= & \sum_{m=-\infty}^{\infty} \sum_{n=m^{*}}^{\infty} s^{m} t^{n} \sum_{K=0}^{n} \frac{(-w)^{K}}{K !(m+K) !(n-K) !}{ }_{L} H^{E_{n-K}[\alpha, m-1]}(0,1, z) .
\end{aligned}
$$

(ii) Setting $s=t=\frac{w}{2}, \alpha=x=0$ and $y=1$ in (4.2), we get

$$
e^{w^{2} z / 4}=\sum_{m=-\infty}^{\infty} \sum_{n=m^{*}}^{\infty}\left(\frac{w}{2}\right)^{m+n} \times \sum_{K=0}^{n} \frac{(-w)^{K}}{K !(m+K) !(n-K) !}{ }_{L} H^{E_{n-K}[\alpha, m-1]}(0,1, z) .
$$

(iii) Setting $s=t=\frac{w}{2}, x=1$ and $\alpha=y=0, z=\frac{2}{w}$ in (3.2), we get a new representation of Tricomi function:

$C_{0}\left(\frac{2}{w}\right)=\sum_{m=-\infty}^{\infty} \sum_{n=m^{*}}^{\infty}\left(\frac{w}{2}\right)^{m+n} \times \sum_{K=0}^{n} \frac{(-w)^{K}}{K !(m+K) !(n-K) !}{ }_{L} H^{E_{n-K}[\alpha, m-1]}\left(1,0, \frac{2}{w}\right)$.

(iv) Taking $m=1, x=1$ and $y=0$ in (4.2), we obtain

$$
\begin{aligned}
& \left(\frac{2}{e^{t}+1}\right)^{\alpha} e^{s-\frac{w t}{s}+z t^{2}} C_{0}(t) \\
= & \sum_{m=-\infty}^{\infty} \sum_{n=m^{*}}^{\infty} s^{m} t^{n} \sum_{K=0}^{n} \frac{(-w)^{K}}{K !(m+K) !(n-K) !}{ }^{L} H^{E_{n-K}[\alpha]}(1,0, z) .
\end{aligned}
$$

(v) Letting $\alpha=m=1$ and $y=0$ in (4.2), we obtain

$$
\begin{aligned}
& \left(\frac{2}{e^{t}+1}\right) e^{s-\frac{w t}{s}+z t^{2}} C_{0}(x t) \\
= & \sum_{m=-\infty}^{\infty} \sum_{n=m^{*}}^{\infty} s^{m} t^{n} \sum_{K=0}^{n} \frac{(-w)^{K}}{K !(m+K) !(n-K) !}{ }^{L} H^{E_{n-K}}(1,0, z) .
\end{aligned}
$$

Acknowledgements. The authors gratefully acknowledge the reviewers for their helpful comments. 


\section{A NEW CLASS OF LAGUERRE-BASED GENERALIZED HERMITE-EULER POLYNOMIALS 99}

\section{REFERENCES}

[1] L. C. Andrews, Special Functions for Engineers and Mathematicians, Macmillan. Co. New York, 1985.

[2] E. T. Bell, Exponential polynomials, Ann. of Math. 35 (1934), 258-277.

[3] L. Comtet, Advanced Combinatorics: The Art of Finite and Infinite Expansions, Reidel, Dordrecht, 1974 (translated from French by J. W. Nienhuys).

[4] G. Dattoli, S. Lorenzutta and C. Cesarano, Finite sums and generalized forms of Bernoulli polynomials, Rendiconti di Mathematica 19 (1999), 385-391.

[5] G. Dattoli and A. Torre, Operational methods and two variable Laguerre polynomials, Atti Accad. Sci. Torino Cl. Sci. Fis. Mat. Natur. 132 (1998), 3-9.

[6] G. Dattoli, A. Torre and A. M. Mancho, The generalized Laguerre polynomials, the associated Bessel functions and applications to propagation problems, Radiation Physics and Chemistry 59 (2000), 229-237.

[7] G. Dattoli, A. Torre and G. Mazzacurati, Monomiality and integrals involving Laguerre polynomials, Rendiconti di Mathematica 18 (1998), 565-574.

[8] G. Dattoli, A. Torre, S. Lorenzutta and C. Cesarano, Generalized polynomials and operational identities, Atti Accad. Sci. Torino Cl. Sci. Fis. Mat. Natur. 134 (2000), 231-249.

[9] H. Exton, A new generating function for the associated Laguerre polynomials and resulting expansions, Jnanabha 13 (1983), 147-149.

[10] A.Erdelyi, W. Magnus, F. Oberhettinger and F. Tricomi, Higher Transcendental Functions, 1-3, McGraw-Hill, New York, 1953.

[11] S. Khan, M. W. Al-Saa and R. Khan, Laguerre-based Appell polynomials: properties and application, Math. Comput. Modeling 52 (2010), 247-259.

[12] N. U. Khan and T. Usman, A new class of Laguerre-based generalized apostol polynomials, Fasc. Math. 57 (2016), 67-89.

[13] N. U. Khan and T. Usman, A new class of Laguerre poly-Bernoulli numbers and polynomials, Advanced Studies in Contemporary Mathematics 27(2) (2017), 229-241.

[14] N. U. Khan and T. Usman, A new class of Laguerre-based poly-Euler and multi poly-Euler polynomials, Journal of Analysis \& Number Theory 4(2) (2016), 113-120.

[15] N. U. Khan, T. Usman and J. Choi, Certain generating function of Hermite-Bernoulli-Laguerre polynomials, Far East Journal of Mathematical Sciences 101(4) (2017), 893-908.

[16] N. U. Khan, T. Usman and J. Choi, A new generalization of Apostol type Laguerre-Genocchi polynomials, C. R. Math. Acad. Sci. Paris Ser. I 355 (2017), 607-617.

[17] B. Kurt, A further generalization of Euler polynomials and on the 2D-Euler polynomials $E_{n}^{2}(x, y)$, Proc. Jangjeon Math. Soc. 15 (2012), 389-394.

[18] P. Natalini and A. Bernardini, A generalization of the Bernoulli polynomials, J. Appl. Math. 3 (2003), 155-163.

[19] M. A. Pathan and Yasmeen, On partly bilateral and partly unilateral generating functions, J. Aust. Math. Soc. 28 (1986), 240-245.

[20] M. A. Pathan and W. A. Khan, Some implicit summation formulas and symmetric identities for the generalized Hermite-Bernoulli polynomials, Mediterr. J. Math. 12 (2015), 679-695.

[21] H. M. Srivastava and H. L. Manocha, A Treatise on Generating Functions, Ellis Horwood Limited, Hichester, 1984.

[22] H. M. Srivastava, M. A. Pathan and M. G. Bin Saad, A certain class of generating functions involving bilateral series, ANZIAM J. 44 (2003), 475-483. 
${ }^{1}$ Department of Applied Mathematics, FACUlty of EngineERING and Technology, Aligarh Muslim University, Aligarh-202002, India

Email address: nukhanmath@gmail.com

${ }^{2}$ Department of Applied Sciences and Humanities, FACULTY OF ENGINEERING AND TECHNOLOGY, Jamia Millia Islamia (A Central University), New Delhi-110025, India Email address: talhausman.maths@gmail.com

${ }^{3}$ Department of Mathematics, FACULTY OF SCIENCE, INTEGRAL UNIVERSITY, LUCKNOW-226026, INDIA

Email address: waseem08_khan@rediffmail.com 